\title{
Schizophrénie, logicité et perspective en première personne.
}

[Schizophrenia, Logicity and First-Person Perspective.]

Manuel Rebuschi ${ }^{1,}$ Maxime Amblard ${ }^{2}$, Michel Musiol ${ }^{3}$

1 Maître de conférences en philosophie, Laboratoire d'Histoire des Sciences et de Philosophie - Archives H. Poincaré (UMR 7117), Université de Lorraine, 91 avenue de la Libération , BP 454 , F-54001 NANCY Cedex. Co-responsable du projet DiaRaFor, Maison des Sciences de I'Homme Lorraine (USR 3261), 91 avenue de la Libération , BP 454 , F-54001 NANCY Cedex

2 Maître de conférences en informatique, Laboratoire Lorrain de Recherche en Informatique et ses Applications (LORIA UMR 7503), Campus faculté des sciences, B.P 239, 54506 VANDOEUVRE-LES-NANCY Cedex

3 Professeur de psychologie, Laboratoire InterPSY - Laboratoire de psychologie de l'interaction et des relations intersubjectives (EA 4432), Université de Lorraine, Campus Lettres et Sciences Humaines, 3 Place Godefroy de Bouillon, BP. 33.97, 54015 NANCY CEDEX. Co-responsable du projet DiaRaFor, Maison des Sciences de I'Homme Lorraine (USR 3261), 91 avenue de la Libération , BP 454 , F-54001 NANCY Cedex 
Résumé : Le délire des schizophrènes est repéré en psychiatrie comme l'une des formes de pensée les plus radicalement déviantes. Il est d'ailleurs appréhendé le plus souvent sur la base de l'analyse des productions verbales (échelles, tests, expérimentation) et du discours des patients. Faut-il considérer que la folie est synonyme de toute perte de logicité, voire de rationalité ? Le supposer aide-t-il à comprendre la schizophrénie ? Dans cet exposé, nous défendrons l'inverse. A partir d'une discussion sur le principe de charité, nous abordons la question de la rationalité et de la logicité des schizophrènes, puis de leur compréhension, dans une perspective philosophique générale. Nous présentons ensuite un travail fondé sur cette hypothèse de logicité, portant sur la formalisation des conversations schizophrènes. Certaines "ruptures" conversationnelles sont manifestement typiques de la pathologie, et leur théorisation soulève la question de leur localisation : faut-il placer les ruptures au plan logico-sémantique, ou bien au plan pragmatique-interactionnel? L'analyse conduit à admettre les deux réponses comme reflétant deux points de vue possibles sur la conversation pathologique: celle, externe ou à la troisième personne, du sujet «normal » et celle, interne ou à la première personne, du sujet schizophrène. Les analyses formelles sont conduites dans le cadre de la Segmented Discourse Representation Theory de Asher \& Lascarides, dont les principes sont succinctement exposés dans l'article.

Abstract: The delirium of schizophrenia is marked in psychiatry as one of the most radically deviant forms of thinking. It is also very often apprehended based on the analysis of verbal productions (scales, tests, experiments) and the speech of patients. Should we consider that madness means a complete loss of logicity or rationality? Does assuming it help to understand schizophrenia? In this paper, we shall defend the reverse. From a discussion on the principle of charity, we address the question of rationality and logicity of schizophrenics and their understanding in a general philosophical perspective. We then present a work, based on this assumption of logicity, on the formalization of 
schizophrenic conversations. Some conversational "failures" are clearly typical of the pathology, and their theoretical account raises the question of their localization: shall one locate the breaks either at a logico-semantic, or at a pragmatic and interactional level? The analysis leads us to admit the two answers as reflecting two possible views on the conversation disease: the external or third person perspective, i.e., that of the "normal" subject, and the internal or first person perspective, specific to the schizophrenic. The formal analysis is conducted within the framework of Segmented Discourse Representation Theory of Asher \& Lascarides, whose principles are briefly outlined in the article.

Mots clefs : contradiction, rationalité, logicité, perspective en première personne, principe de charité, logique paraconsistante, pragmatique, schizophrénie.

Key words: Contradiction, Rationality, Logicity, First-Person perspective, Principle of charity, Paraconsistent Logic, Pragmatics, Schizophrenia 


\section{Introduction}

Peut-on comprendre la folie ? Dans les cas les plus radicaux comme le délire des schizophrènes, cela paraît impossible lorsqu'on se réfère aux canons classiques de la rationalité : le déni de réalité et les pensées apparemment contradictoires caractéristiques de cette pathologie suggèrent de renoncer. II faudrait alors se contenter d'une approche purement externe, à la troisième personne, de la vie mentale des fous.

Nous allons argumenter contre cette impossibilité. Sans nier la pertinence des explications à la troisième personne (de type neurobiologique, psychanalytique, ou autre), nous refusons l'hypothèse réductionniste suivant laquelle ces explications permettraient de saisir le phénomène de la folie dans son intégralité. Nous pensons pouvoir abonder en faveur de l'hypothèse selon laquelle la folie n'exclut pas la rationalité du sujet, même si celle-ci est déviante, et que par voie de conséquence, un point de vue à la première personne sur la pathologie est défendable.

Notre conception s'appuie sur un travail empirique d'analyse de corpus issus de conversations pathologiques. Les conversations recueillies, entre un patient schizophrène et un psychologue, sont le lieu de discontinuités manifestes pour un interlocuteur normal (i.e., non pathologique). Une analyse pragmatique de type hiérarchique et fonctionnelle permet le repérage des discontinuités pertinentes dans ces corpus, et de sélectionner des fragments sur lesquels une analyse plus formelle est conduite. Nous construisons alors une représentation conversationnelle en S-DRT (Segmented Discourse representation theory), qui permet de formaliser simultanément les aspects sémantiques et pragmatiques du dialogue.

Ce travail d'analyse, conduit dans un cadre interdisciplinaire, soulève plusieurs questions philosophiques: La logique peut-elle tolérer les contradictions ? A 
défaut, la rationalité peut-elle tolérer les contradictions ? La non-contradiction est-elle une précondition de la compréhension d'autrui ? Dans le présent article nous voulons aborder ces questions à partir d'une discussion autour du principe de charité. Notre discussion est sous-tendue par un questionnement épistémologique relatif à la formalisation des conversations : faut-il envisager une ou deux représentations conversationnelles pour une conversation pathologique ? Et surtout : où faut-il placer les incohérences du schizophrène ?

La suite de l'article est organisée en quatre sections. Dans la section 2, nous traitons spécifiquement de questions philosophiques : le principe de charité et son rapport à la compréhension. Nous défendrons une version forte, quinienne, du principe, y compris dans la perspective d'une compréhension des schizophrènes. Dans la section suivante, 3, nous présentons les principes théoriques et épistémologiques qui président à nos analyses formelles des conversations pathologiques. Dans la section 4, nous exposons brièvement quelques données relatives au corpus, et un exemple, avant de conclure l'article.

\section{Interprétation et charité}

\section{Le principe de charité}

Quine [1], et dans son sillage Davidson [2], ont défendu la nécessité du principe de charité pour l'interprétation d'autrui. L'idée est de maximiser la vérité des croyances de l'autre, mais aussi et surtout de supposer leur consistance, i.e. leur non-contradiction logique. Derrière le principe de charité se cachent en fait plusieurs variantes, que nous pouvons citer de la plus forte à la plus faible : 
- Un postulat de logicité forte : l'interprété se conforme à la logique classique $^{1}$;

- Un postulat de logicité faible : l'interprété se conforme au principe de contradiction (il n'admet pas simultanément $A$ et non-A) ;

- Un postulat de rationalité : l'interprété est rationnel.

On peut évidemment défendre le principe de contradiction sans pour autant adopter la logique classique ${ }^{2}$, d'où les appellations de versions forte et faible du postulat de logicité. Suivant le postulat de logicité dans sa version faible, le sujet respecte le principe de contradiction mais rien n'est dit quant à sa logique générale qui peut être non classique. Le postulat de rationalité est quant à lui relativement indépendant du postulat de logicité, puisque l'on peut envisager un sujet qui ne respecterait pas le postulat de logicité même dans sa version faible, donc qui ne respecterait pas le principe de contradiction, mais qui serait néanmoins rationnel.

Quine a défendu la nécessité du principe de charité comme postulat de logicité forte dans un contexte de "traduction radicale", à savoir la situation hypothétique et idéale d'un anthropologue arrivant au milieu d'un peuple n'ayant eu aucun contact à l'extérieur :

Supposons qu'on prétende que certains indigènes sont disposés à accepter comme vraies certaines phrases traduisibles dans la forme "p et non-p". Cette supposition est absurde au regard de nos critères sémantiques. (...) Une traduction [perverse] peut rendre les locutions indigènes aussi étranges que

\footnotetext{
${ }^{1}$ Nous ne suggérons pas que les sujets raisonnent comme on fait des déductions dans un calcul logique, mais que leurs raisonnements tendent à se conformer à telles ou telles normes logiques.

${ }^{2}$ La plupart des logiques non classiques (logique de la pertinence, logique intuitionniste, etc.) conservent le principe de contradiction.
} 
I'on veut. Une meilleure traduction leur imposera notre logique (...). ([1], §13, p.99)

Mais ce que vise le principe, c'est un rapatriement at home : la traduction (i.e. I'interprétation au cours de laquelle nous projetons nos propres hypothèses) ne vise pas tant les Indiens de telle ou telle tribu que nos congénères, qui parlent apparemment la même langue que nous ${ }^{3}$ :

Qu'une bonne traduction préserve les lois logiques, cela est implicite partout où, pour nous exprimer paradoxalement, aucune langue étrangère n'entre en scène. Ainsi lorsqu'à nos questions concernant une phrase française un locuteur français répond "Oui et non", nous présumons que la phrase qui fait l'objet de la question est comprise différemment pour l'affirmation et pour la négation ; cela plutôt que de croire que le locuteur est assez stupide pour affirmer et nier la même chose. (...) [L]a stupidité de notre interlocuteur, audelà d'un certain point, est moins probable qu'une mauvaise traduction - ou, dans le cas domestique, qu'une divergence linguistique. ([1], pp. 100-101)

L'interprétation, et la possibilité d'interprétations rivales et incompatibles, se posent également dans ce cas plus familier puisque, pour le dire vite, nous ne disposons pas de faits (empiriques) pour trancher diverses questions sur le sens que veulent nous communiquer nos interlocuteurs. Selon Davidson, postuler la rationalité et la logicité est ainsi une pré-condition de la compréhension d'autrui :

Crediting people with a large degree of consistency cannot be counted mere charity: it is unavoidable if we are to be in a position to accuse them meaningfully of error and some degree of irrationality. Global confusion, like universal mistake, is unthinkable, not because imagination boggles, but because too much confusion leaves nothing to be confused about and massive error erodes the background of true belief against which alone failure can be

\footnotetext{
${ }^{3}$ Pour une présentation du principe de charité, voir [3]. Pour une discussion critique, voir [4].
} 
construed. (...) To the extent that we fail to discover a coherent and plausible pattern in the attitudes and actions of others we simply forego the chance of treating them as persons. ([2], pp. 221-222)

La justification du principe de charité n'est plus alors seulement méthodologique, autrement dit le principe n'est pas seulement rendu indispensable pour l'interprétation : c'est aussi une justification conceptuelle, au sens où la rationalité est ici conçue comme constitutive des (concepts de) croyances vraies et autres attitudes des sujets (cf. [4]).

\section{Comprendre les fous}

La question est alors : que se passe-t-il quand nos interlocuteurs sont fous?

Très souvent, ce que disent les fous n'est pas considéré comme sérieux, mais cela n'est pas non plus considéré comme devant être pris au sérieux pour avoir une bonne analyse de la folie. Les conceptions dominantes sont en effet réductionnistes. La folie doit pouvoir s'expliquer complètement par un dysfonctionnement du cerveau (réductionnisme neurobiologique, voire génétique), ou par les strates du subconscient (réductionnisme psychanalytique). On reste alors contraint à un point de vue externe, à la troisième personne, du sujet. L'analyse visée est celle d'une explication causale, et s'il y a bien rationalisation de la folie par l'analyse, la seule rationalité à l'œuvre est celle du psychologue : on nie toute rationalité au fou.

Le psychologue et philosophe américain Louis A. Sass $[5,6]$ conteste ces approches réductionnistes et défend la perspective d'une analyse qui prenne en compte le point de vue interne, à la première personne : il s'agit bien non seulement d'expliquer mais aussi de comprendre en termes de raisons ce qui anime le sujet fou. Cela signifie reconnaître une rationalité du sujet étudié, à l'inverse de ce qui semble ressortir des critères de diagnostics en psychiatrie (cf. la critique du DSM-IV par Henriksen [7]). 
Insistons sur le fait que cette approche en première personne, historiquement revendiquée pour les "sciences de l'esprit» par Dilthey, est parfaitement compatible avec les explications à la troisième personne offertes par la neurobiologie et/ou la génétique, dominantes en psychiatrie. II ne s'agit pas pour nous de statuer ici sur la valeur de ces dernières, ni sur la classification des pathologies synthétisée dans le DSM. C'est bien le réductionnisme qui est en cause : nous contestons le fait que l'appréhension de la pathologie puisse être entièrement prise en charge par les explications à la troisième personne.

Comprendre les fous suppose de saisir leur rationalité, mais la rationalité des fous est déviante. Où placer la déviance ? On va s'intéresser en particulier à la schizophrénie, pathologie donnant lieu aux délires ou incohérences réputés les plus radicalement déviants. En parlant de rationalité des fous, et plus spécialement des schizophrènes, on admet donc une partie du principe de charité. Jusqu'où peut-on aller?

Les schizophrènes sont apparemment contradictoires. C'est ce qui ressort de l'analyse de corpus extraits de conversations schizophrènes : il y a des ruptures ou discontinuités conversationnelles fréquentes. Dans certains cas, ces ruptures conversationnelles correspondent à des moments où, manifestement, le sujet schizophrène paraît accepter (et même engendrer) des jugements contradictoires. Comment rendre compte de cela?

\section{Localisation des incohérences : au niveau du contenu sémantique}

Si l'on suit Quine et sa conception du principe de charité, où rationalité rime avec logique classique (du premier ordre), c'est-à-dire où la charité est conçue 
comme un postulat de logicité forte, on aurait le choix entre ${ }^{4}:(1)$ considérer que le principe de charité n'est pas valable dans le cas des schizophrènes, mais c'est alors leur dénier toute rationalité et être renvoyé aux approches réductionnistes citées plus haut, donc renoncer à les comprendre; et (2) considérer que le principe de charité s'applique pleinement, autrement dit que les sujets sont malgré tout logiques et même classiques, mais que les schizophrènes ne comprennent pas le sens des mots comme nous et que nous ne disposons pas du bon manuel de traduction entre leur langage et le nôtre, ce qui rend leur compréhension également impossible pour nous.

On peut contester l'appréhension de la charité par Quine et suivre Graham Priest [8], pour lequel les postulats de logicité (forte ou faible) et de rationalité doivent être dissociés. Le sujet schizophrène serait alors rationnel, mais il ne serait pas logique au sens de la logique classique, ni même simplement au sens où il respecterait le principe de contradiction. II aurait une autre logique, une logique paraconsistante, tolérante aux contradictions. Cela rendrait compte du fait que le délire structuré paraît bien avoir un sens pour le sujet (en première personne), même si nous (en troisième personne) considérons que c'est du délire, donc éventuellement une pensée non logique : la dualité des points de vue serait une dualité de logiques. Priest serait certainement très insatisfait d'apprendre que la logique paraconsistante est réservée à la pensée des schizophrènes, mais c'est accessoire pour notre propos. Ce qui l'est moins et que l'on va discuter, c'est qu'en suivant cette position on en vient à assumer des logiques différentes entre les fous et les non fous, en tout cas quand ils conversent ensemble : ce qui conduit également à considérer que nous, les non fous, ne pouvons pas comprendre les fous, simplement parce que nous n'avons pas la même logique qu'eux.

\footnotetext{
${ }^{4}$ II ne s'agit pas ici de considérer les positions qu'aurait pu défendre Quine, mais uniquement de voir quelles positions sont compatibles avec sa conception « forte » du principe de charité.
} 
Dans son étude du principe de contradiction chez Aristote, Łukasiewicz [9] défendait l'idée que la version psychologique du principe de contradiction devrait être empiriquement validée ou réfutée, et non pas prouvée a priori. La version psychologique du principe de contradiction, c'est l'impossibilité d'avoir des croyances (des "convictions ») contraires, autrement dit l'impossibilité d'avoir des croyances dont les contenus sont des jugements contradictoires (du type $A$ et non-A). Le cas des schizophrènes montrerait ainsi à peu de frais, en ce qui les concerne tout au moins, que le principe psychologique de contradiction ne tient pas.

Mais c'est aller un peu vite en besogne. Łukasiewicz en 1910 s'appuie sur une épistémologie naïve : pour qu'il y ait détermination " empirique » vs. a priori de la validité du principe, il faut supposer qu'il y a des sortes de faits psychologiques bruts. Mais en psychologie pas plus qu'ailleurs il n'y a de faits bruts : les faits psychologiques sont théoriquement chargés, c'est-à-dire que les données sont interprétées en fonction de nos hypothèses théoriques. Devant le phénomène de la contradiction apparente, la question de la localisation de I'inconsistance demeure entière.

\section{Localisation des incohérences : dans la présentation du contenu}

Dans la lignée d'autres théoriciens, Louis Sass [5] conteste que l'épreuve de réalité déficitaire, habituellement intégrée parmi les symptômes de la schizophrénie, caractérise adéquatement la pensée des sujets. L'épreuve de réalité déficitaire est cette imperméabilité au réel qui se traduirait par la production de croyances fausses et même contradictoires au sujet du réel. Ce que conteste Sass, avec d'autres, c'est de faire porter le déficit sur le contenu (propositionnel) des états mentaux, alors que l'on pourrait (et devrait) bien plutôt considérer que le défaut porte sur les états eux-mêmes, c'est-à-dire sur le mode d'appréhension de ce contenu. Selon Sass, là où nous voyons des 
croyances, le sujet schizophrène dispose d'états d'un type bien moins engagé vis-à-vis du réel. Pour Campbell [10], il s'agit d'hypothèses-cadre, que l'on peut rapprocher des capacités d'arrière-plan de Searle [7].

Sass rapproche quant à lui l'attitude mentale des schizophrènes de celle qui est, selon Wittgenstein, sous-jacente au solipsisme philosophique - ce qui n'est pas sans évoquer la célèbre citation de Shopenhauer : "Le solipsiste est un fou enfermé dans un blockhaus imprenable "! L'idée est que loin d'objectiver les contenus de ses attitudes (que nous appellerons schizo-croyances), le sujet schizophrène aurait plutôt tendance à les subjectiviser, c'est-à-dire à leur dénier tout statut véridique. Cela rejoint une mise en question généralisée des perceptions à la manière du scepticisme radical qui sous-tend le solipsisme. Les pensées délirantes et les états issus des perceptions sont traités sur le même mode, comme des schizo-croyances et non comme des croyances ${ }^{5}$.

En quoi jouer sur le contenant, sur le type d'état mental, permet-il d'évacuer les contradictions du contenu ? C'est délicat à décrire ici puisque les schizocroyances sont caractéristiques de la pensée schizophrène, et donc que nous ne sommes pas censés en posséder : c'est un type d'état mental que les non schizophrènes n'ont pas, ce qui explique la difficulté de comprendre (p.ex. par empathie) les sujets schizophrènes ${ }^{6}$.

\footnotetext{
${ }^{5}$ Il est notable que ce point paraisse converger avec certaines approches très formelles de la contradiction, chez les logiciens paraconsistants. Villadsen [11] propose ainsi une analyse paraconsistante des assertions dont le principe consiste à suspendre son jugement sur une assertion (en lui attribuant une valeur de vérité indéterminée) pour permettre à un ensemble d'assertions contradictoires de coexister (art.cit. p.106).

${ }^{6}$ L'idée que la compréhension passe par l'empathie sous-tend l'alternative proposée par Bonnay et Cozic [4] au principe de charité. Selon eux, les sciences cognitives suggèrent que la compréhension d'autrui repose principalement sur des mécanismes de simulation. Dans le cas de la schizophrénie, l'étrangeté du sujet à interpréter est telle que même la simulation ne peut pas fonctionner. On voudrait donc défendre ici une conception de la compréhension en première personne qui ne passe ni par l'empathie ni par la simulation.
} 
On peut toutefois donner une illustration de la question à l'aide d'un type d'état mental déviant que l'on ne trouve nulle part, mais construit sur mesure, et qui générerait des contradictions au niveau du contenu là où le type d'état mental «normal » correspondant n'en provoquerait pas. Ce type d'état, appelons-le "imajourner»: c'est un état qui dure une journée, et qui correspond à l'imagination continue dans le temps, disons jusqu'à la prochaine phase de sommeil. Si j'imajourne maintenant qu'il pleut, alors cela signifie que j'imagine qu'il pleut jusqu'à ce soir. Alors si j'imajourne qu'il pleut, et qu'une minute après j'imajourne qu'il ne pleut pas, j'entretiendrai des états (des imajournations) dont le contenu est contradictoire. Tandis que si je n'imajourne rien mais que j'imagine, alors je peux imaginer qu'il pleut, une minute plus tard imaginer qu'il ne pleut pas, et je n'entretiendrai pas d'état (du type de l'imagination) dont le contenu serait contradictoire. En bref : le type d'état en cause est crucial pour déterminer si le contenu de deux états est ou non contradictoire. De même que les mêmes contenus imaginés ne produisent pas de contradiction alors qu'ils en produisent une lorsqu'ils sont imajournés, il faut concevoir que les mêmes contenus, contradictoires quand ils sont les contenus de croyances, cessent d'être contradictoires lorsqu'ils sont les contenus de schizo-croyances.

\section{Vers l'analyse des conversations}

\section{Incohérence du contenu, ou incohérence pragmatique ?}

La stratégie que nous développons pour l'analyse des conversations ne s'appuie pas sur une nouvelle typologie des états mentaux. Mais là où nous nous rapprochons de conceptions comme celle développée par Sass, c'est que nous considérons également que le problème de la pensée schizophrène, telle qu'elle s'exprime dans la conversation, n'est pas un problème d'inconsistance 
du contenu. Nous postulons le parfait classicisme logique des interlocuteurs schizophrènes, et donc nous admettons que la version forte (à la Quine) du principe de charité s'applique également à leur $\operatorname{cas}^{7}$. Mais nous plaçons la déviance de la rationalité dans les règles d'usage du langage, dans les conventions linguistiques de type rhétorique et pragmatique.

Ce qui ressort de cette approche se distingue de l'idée de Brunet [12] que la séparation nette entre raisonnement (processus mettant en mouvement des états) et argument (liant logiquement des contenus) doit être calquée sur la distinction entre, respectivement, point de vue à la troisième personne et point de vue à la première personne. Selon notre conception, le point de vue à la première personne, qui vise à rendre compte de la rationalité de la pensée d'un sujet, ne peut pas se réduire à la seule évaluation du contenu : la manière dont ce contenu est structuré (par tel ou tel type d'état mental dans une approche du délire à la Sass, par telles ou telles relations pragmatiques dans l'analyse des conversations pathologiques que nous développons), cette manière constitue un composant essentiel de la rationalité. En bref, la rationalité ne se réduit pas à la logicité.

Nos analyses empiriques portent sur des transcriptions de conversations entre un sujet schizophrène et un sujet « normal » (non schizophrène), psychologue. Les conversations donnent lieu à des ruptures qui sont perçues comme telles par le sujet normal, mais pas nécessairement par l'interlocuteur schizophrène à l'origine de ces ruptures. Les analyses consistent en la construction de représentations conversationnelles suivant le formalisme de la S-DRT, succinctement présenté plus bas. Ces représentations comprennent deux niveaux : une représentation sémantique (le contenu de la conversation), et

\footnotetext{
${ }^{7}$ Egalement, c'est-à-dire ni plus ni moins qu'au non schizophrène : une discussion générale sur le statut de la logique n'est évidemment pas notre propos dans cet article.
} 
une représentation pragmatique (la structure hiérarchique des actes de langage qui constituent la conversation).

Pour l'analyse des conversations pathologiques, nous proposons systématiquement la construction simultanée de deux représentations conversationnelles, une par interlocuteur. Du côté du sujet schizophrène, conformément au principe de charité la représentation est dépourvue de contradictions au niveau sémantique; s'il y a des ruptures, celles-ci interviennent au niveau pragmatique, par l'infraction de règles de construction des arbres de la S-DRT. La situation n'est pas la même en face. Dans les conversations qui constituent les corpus étudiés, l'interlocuteur normal est un psychologue en charge de poursuivre l'entretien: il fait ainsi en sorte de " réparer » la structure conversationnelle après une rupture, même si cette dernière est telle qu'elle aurait provoqué l'interruption d'une conversation analogue dans une autre situation. On a alors un postulat correspondant à cette prescription, à savoir la construction d'une représentation respectant les contraintes pragmatiques; cette option entraîne l'apparition d'inconsistances au niveau sémantique.

La dualité des représentations conversationnelles reflète ainsi la dualité des points de vue sur la conversation: le sujet schizophrène est apparemment contradictoire pour le sujet normal, si bien que la conversation fonctionne mais que la représentation du monde co-construite est inconsistante (point de vue à la troisième personne); à l'inverse, le dysfonctionnement du sujet schizophrène résidant dans sa gestion des relations pragmatiques, la représentation du monde construite par la conversation ne souffre pas de ce défaut (point de vue à la première personne). La situation résultante est résumée dans le Tableau 1. 
Tableau 1. Dualité de points de vue sur la conversation

\begin{tabular}{|c|c|}
\hline $\begin{array}{c}\text { Sujet normal } \\
\text { (à la } 3^{\mathrm{e}} \text { personne) }\end{array}$ & $\begin{array}{c}\text { Schizophrène } \\
\text { (à la } 1^{\mathrm{e}} \text { personne) }\end{array}$ \\
\hline $\begin{array}{c}\text { correction pragmatique } \\
\text { déviance sémantique }\end{array}$ & cóviance pragmatique \\
\hline Contenu contradictoire : & Contenu cohérent : \\
apparence de contradiction & tout va bien ! \\
\hline
\end{tabular}

\section{Présentation (rapide) de la S-DRT}

Le cadre formel utilisé est celui de la S-DRT [13]. II s'agit de combiner deux niveaux d'analyse pour pouvoir rendre compte des processus interprétatifs à l'œuvre dans les conversations : l'analyse du contenu sémantique et l'analyse pragmatique conversationnelle. La première est prise en charge par les Structures de représentation de discours segmenté (S-DRS) inspirées des DRS de la DRT, qui sont une construction syntaxique mise à jour au fil du flux conversationnel [14]. La conversation suppose en outre la mise en place de relations pragmatiques entre actes de langage des interlocuteurs (à la fois au niveau intra-locuteur et interlocuteurs), dont la complexité donne lieu à une structuration hiérarchique appréhendée dès les années 1980 en linguistique [15]. Nous proposons de formaliser ces relations à l'aide des relations rhétoriques de la S-DRT. Une conversation est alors interprétée par une double construction : celle d'un arbre hiérarchique reliant les actes, et celle des S-DRS représentant le contenu sémantique des actes.

L'hypothèse que nous faisons est que l'interlocuteur schizophrène ne se conforme pas toujours aux règles devant prévaloir lors de cette double 
construction, ce qui explique le phénomène de rupture conversationnelle perçu par l'interlocuteur normal [16-18].

Les structures rhétoriques de la S-DRT relient les interventions des locuteurs; elles sont représentées sous forme d'arbres hiérarchiques, avec des relations verticales, horizontales ou obliques selon le type. Une narration sera ainsi typiquement une relation horizontale (de même niveau hiérarchique), de même que la réponse à une question, tandis qu'une élaboration sera une relation verticale (subordonnée à ce qu'elle élabore), et une question une relation oblique (verticale donc subordonnée, mais horizontale parce qu'appelant une réponse).

Un exemple de structure S-DRT, repris et légèrement modifié de [19], est représenté sur la Figure 1 . Cet exemple correspond à un cas de discours monologique, mais ce que l'on y voit vaut pour les cas de dialogue. L'arbre est mis à jour au fil de la conversation (ou du discours). Chaque intervention ultérieure de l'un des interlocuteurs (respectivement : du locuteur) est censée se rattacher à la représentation conversationnelle (respectivement: du discours) déjà construite. La structuration permet alors de dégager des contraintes générales affectant les sites de rattachement. La contrainte principale est la règle dite de la frontière droite, qui force le rattachement aux nœuds se situant à droite de l'arbre. Ainsi à partir de l'exemple donné cidessus, l'assertion de la phrase "II l'a trouvé vraiment merveilleux » serait ambiguë, le pronom «le» («l'») pouvant prendre plusieurs valeurs. Ce qu'indique la règle de la frontière droite, c'est que cette phrase ne peut pas être rattachée à "II a mangé du saumon » et donc que "le " ne peut pas renvoyer au saumon, mais qu'elle peut être rattachée à n'importe quel autre 
nœud de l'arbre, et donc que « le " peut renvoyer au fromage, au repas, ou au moment de la veille au soir ${ }^{8}$.

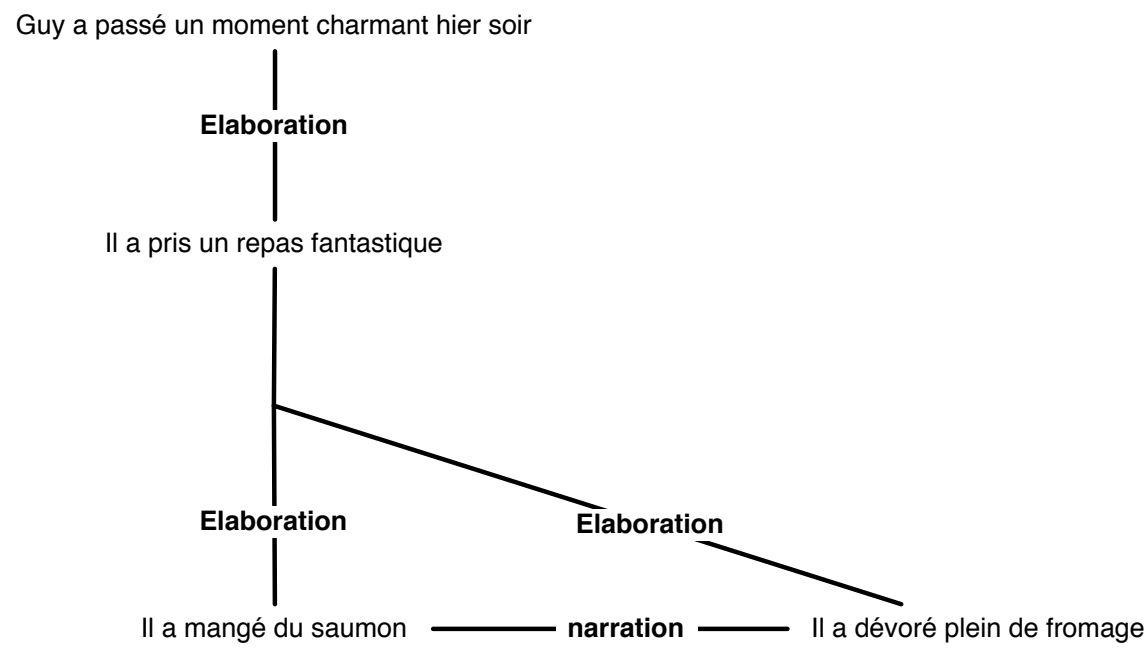

Figure 1. Un exemple de structure de S-DRT

\section{Spécificités de la formalisation des conversations schizophrènes}

Nous faisons une double conjecture : 1) les schizophrènes sont logiquement consistants ; par conséquent les ruptures interviennent au niveau du processus de construction de la représentation conversationnelle sur la dimension pragmatique (sur les relations rhétoriques des S-DRS) ; 2) La sous-spécification ${ }^{9}$ joue un rôle central dans ces ruptures, ce qui pourrait se résumer par le slogan : Un choix n'est jamais définitif! La première conjecture n'est autre que "l'implémentation » attendue du postulat de logicité forte. La seconde conjecture, qui correspond en premier lieu à un constat empirique [17], est une heuristique permettant la localisation des stratégies réparatrices à l'œuvre de la part du locuteur normal : quand il y a apparence de discontinuité, ce dernier

\footnotetext{
${ }^{8}$ La théorie de la S-DRT introduit également des variables représentant la conjonction des élaborations. Ainsi la frontière droite permet d'accéder à l'énoncé contenant le saumon, mais pas au saumon lui-même.

${ }^{9}$ Ambiguïté d'ordre syntaxique, sémantique ou pragmatique, appelée à être discriminée en contexte discursif.
} 
utilise les relations sous-spécifiées pour maintenir la cohérence pragmatique de la S-DRS - autrement dit, la flexibilité des relations sous-spécifiées permet de construire une représentation conversationnelle quoi qu'il arrive.

Tableau 2. Types de relations pragmatiques repérées

\begin{tabular}{|c|c|c|}
\hline Relations horizontales & Relations verticales & Relations obliques \\
\hline Narration & Elaboration & Question \\
Réponse phatique & Elaboration : explication & Question : conduite \\
Suite et illustration & Elaboration : prescription & Méta-question \\
& Phatique & Conduation \\
& & Contre-élaboration \\
& & Justification \\
\hline
\end{tabular}

Nous avons dû étendre l'ensemble de base des relations rhétoriques de la SDRT pour rendre compte de la complexité de l'interaction dialogique, et notamment prendre en compte les ajustements méta-conversationnels absents de la théorie originelle. L'étude des corpus nous a conduits à identifier les types de relations suivants récapitulés dans le Tableau 2 .

Outre la typologie des relations rhétoriques, nous introduisons le critère thématique: le thème conversationnel est l'élément sémantique le plus pertinent pour rendre compte des discontinuités à l'œuvre dans les conversations pathologiques. Chaque séquence conversationnelle est en effet articulée autour d'un thème, qui constitue notamment le principal élément contextuel permettant de désambiguïser les expressions sous-spécifiées. Le thème conversationnel change généralement après un signal conventionnel (p.ex. : " Et sinon... », ou " Par ailleurs... »), ou après une autre forme de clôture 
d'une séquence conversationnelle - par exemple, lorsqu'il y a complétude interactionnelle. Le maintien du thème courant permet ainsi la continuation d'une arborescence, tandis que le changement de thème suppose une remontée dans l'arbre pour un rattachement à un nœud dominant correspondant à une séquence antérieure de l'échange. Notre formalisation introduit des boîtes thématiques, qui sont des ensembles cohérents d'unités de discours pouvant être mutuellement incluses (mais sans chevauchement) ${ }^{10}$, ainsi que la règle de remontée dans un arbre S-DRT, qui n'est autorisée que si la boîte thématique courante est correctement fermée.

Enfin, bien que la S-DRT soit une théorie du discours et non du dialogue, nous supposons que dans les entretiens, le sujet « normal » intervient pour soutenir l'échange et non en tant que participant usuel. Ainsi, ces dialogues servent à rendre compte de l'expression de la pensée du schizophrène et sont vus comme des discours.

\section{Corpus étudié et résultats}

\section{Quelques données issues du corpus}

Les conversations pathologiques sont des interactions verbales entre un patient schizophrène et un interlocuteur normal. Les études de la schizophrénie basées sur l'analyse pragmatique des conversations ont révélé quatre types de discontinuités, résumées dans le Tableau 3. Les discontinuités détectées peuvent être attribuées au comportement discursif et dialogique du patient. Elles reflètent, sur un plan pragmatique et formel, des comportements

\footnotetext{
${ }^{10}$ Etant donné qu'il relève du niveau sémantique, le critère thématique pourrait en principe être représenté par un marqueur à l'intérieur de la S-DRS. Comme il s'agit du seul élément sémantique appelé à figurer dans nos représentations simplifiées, nous avons fait le choix de renoncer aux S-DRS pour ne laisser que l'arborescence pragmatique, les boîtes thématiques encadrant des ensembles de nœuds.
} 
incongrus voire incohérents susceptibles de se manifester chez les sujets schizophrènes.

Tableau 3. Quatre types de discontinuités conversationnelles

\begin{tabular}{r|c|c} 
& Simple & Complexe \\
\hline proximale) & intervention & \\
\hline Triadique (et distale) & Rupture de l'échange & Rupture de l'intervention \\
& & complexe
\end{tabular}

Les différents types de ruptures sont comparés empiriquement en fonction des spécificités diagnostiques des syndromes "paranoïde " et " désorganisé ". Le corpus de départ comprend 30 entretiens avec 14 patients schizophrènes "paranoïdes » $(\mathrm{SCH}-\mathrm{P}), 8$ patients schizophrènes " désorganisés » $(\mathrm{SCH}-\mathrm{D})$ et 8 sujets dans un groupe de contrôle (CTR). Au total, 403 séquences d'interactions ont été obtenues: plus de $30 \%$ présentant des discontinuités chez les schizophrènes, contre moins de $2 \%$ pour le groupe de contrôle. Fait notable, toutes les discontinuités triadiques (au nombre de 9), également qualifiées de décisives et comportant des contradictions apparentes, sont apparues dans le sous-corpus des schizophrènes paranoïdes (SCH-P) [20]. Ce comportement linguistique spécifique peut être interprété comme le signe d'une perturbation sous-jacente du processus par lequel les schizophrènes comprennent et « calculent » les intentions communicatives de leurs interlocuteurs.

\section{Un exemple d'analyse pragmatico-sémantique}

Un exemple de rupture est offert dans l'extrait suivant [21], qui donne un aperçu de ce que peuvent être les dysfonctionnements observés chez les 
schizophrènes ( $A$ est le psychologue, $B$ le patient ; les flèches entre parenthèses sont de simples indices phonétiques) :

B124 : ${ }^{1}$ Oh ouais $(\uparrow)$ et pis compliqué $(\downarrow)$ et c'est vraiment très très compliqué $(\rightarrow)^{2}$ la politique c'est quelque chose quand on s'en occupe faut être gagnant parce qu'autrement quand on est perdant $c^{\prime}$ est fini quoi $(\downarrow)$

A125 : oui

B126 : J. C. D. est mort, L. est mort, P. est mort euh (...)

A127 : Ils sont morts parce qu'ils ont perdu à votre avis ( $\uparrow)$

B128 : ${ }^{1}$ Non ils gagnaient ${ }^{2}$ mais si ils sont morts, c'est la maladie quoi c'est c'est $(\rightarrow)$

A129 : Ouais c'est parce qu'ils étaient malades, c'est pas parce qu'ils faisaient

de la politique $(\uparrow)$

B130 : Si enfin $(\rightarrow)$

A131 : Si vous pensez que c'est parce qu'ils faisaient de la politique ( $\uparrow)$

B132 : ${ }^{1}$ Oui tiens oui ${ }^{2}$ il y a aussi C. qui a accompli un meurtre là $(\rightarrow)$ il était présent lui aussi qui est à $B .{ }^{3}$ mais enfin $(\rightarrow)$ c'est encore à cause de la politique ça

La représentation de l'extrait selon le schizophrène (B) est donnée dans la Figure 2. L'intervention B126 ouvre une nouvelle boîte thématique passant de 
la mort symbolique à la mort réelle sans poser de problème, la première partie de la dérivation ayant été correctement close. La dérivation se poursuit jusqu'à I'intervention B130 qui est ambiguë et dont le contenu sémantique est faible. Quel que soit le choix fait, pour le rattachement, l'intervention B132 résout l'ambiguïté. Et c'est là que se pose un premier problème. La contrainte de la frontière droite sur cette structure ne permet pas le rattachement de B130 sur A125 (mais uniquement sur A129, B128 ou B126) et pourtant, c'est en ce point que B130 doit thématiquement être rattachée. Nous avons une première discontinuité. Un problème similaire se pose sur l'intervention $B^{2} 132$ qui cette fois doit arbitrairement être rattachée sur la seconde boîte thématique, ce qui n'est pas possible. La Figure 2 présente ainsi la représentation conversationnelle par le patient schizophrène, avec respect de la cohérence thématique, c'est-à-dire sémantique, et incohérence au niveau des relations pragmatiques.

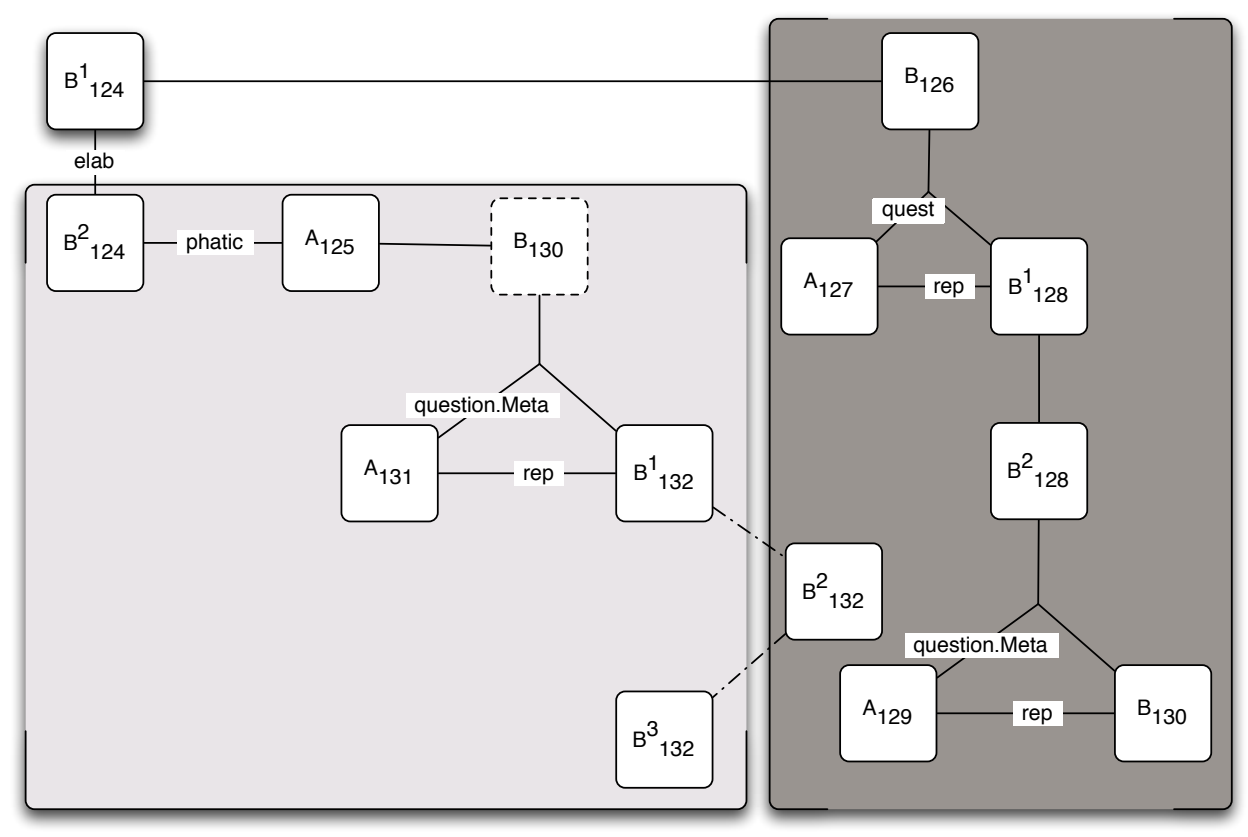

Figure 2. Représentation S-DRT de la séquence conversationnelle attribuée au sujet schizophrène 


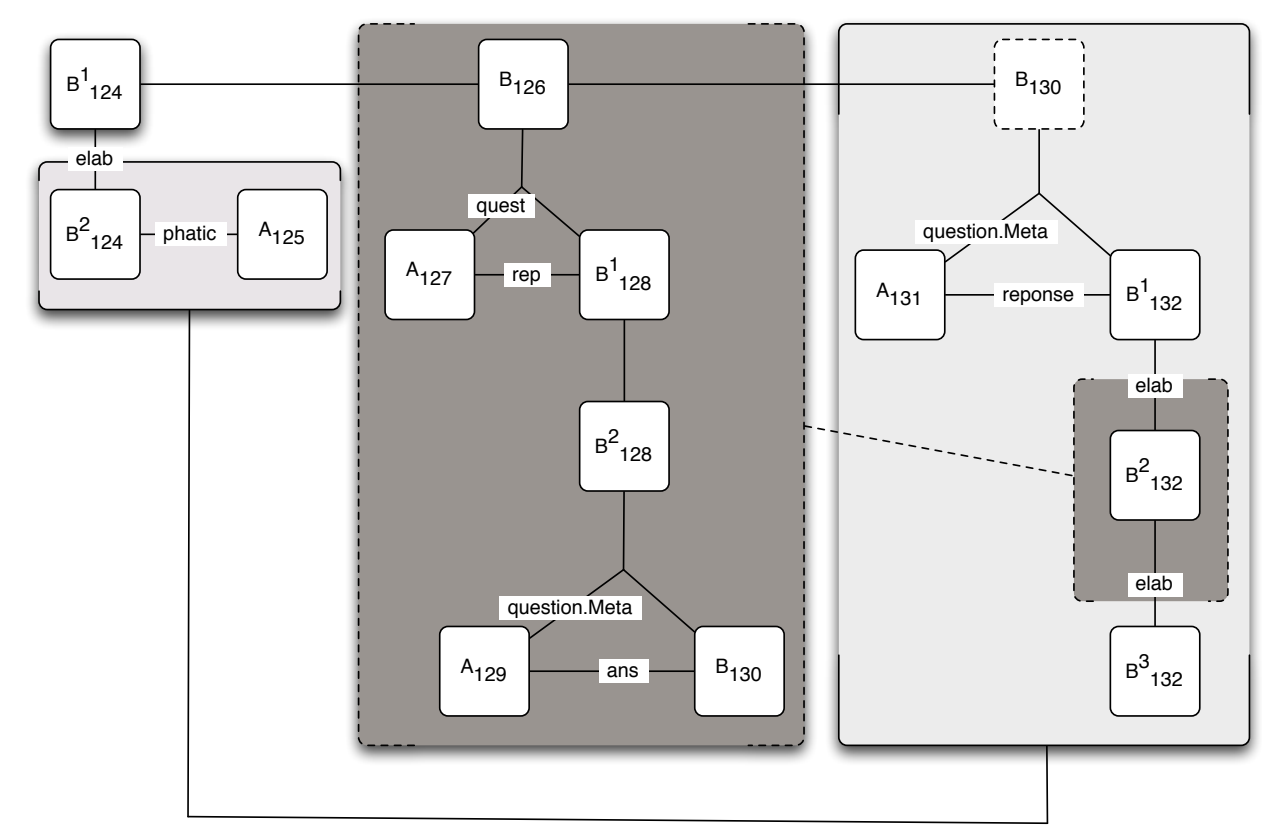

Figure 3. Représentation S-DRT de la séquence conversationnelle attribuée au psychologue

La représentation de la même conversation par le psychologue, Figure 3, est tout à fait semblable au début de la conversation. Elle diverge au niveau de l'intervention B130, qui par respect de la cohérence pragmatique, ne peut pas être rattachée en A125 : I'intervention B130 est dédoublée chez le psychologue comme chez le schizophrène, mais conformément à la règle de remontée et à la contrainte de la frontière droite, B130 est ensuite rattachée à B126. Par sa question en $\mathrm{A} 131$, le psychologue interroge en effet à nouveau l'affirmation B126 du patient. On est alors en présence, sinon d'une incohérence sémantique, du moins d'une faiblesse, puisqu'il y a reprise de la thématique initiale tandis que celle-ci est close depuis A125. L'incohérence sémantique est en revanche manifeste en $B^{2} 132$, quand le patient revient sans préavis à la thématique précédente close en $\mathrm{B} 130$, puis revient à la thématique courante en $B^{3} 132$. Du point de vue du sujet normal (Figure 3 ), on est donc en présence 
d'inconsistances sémantiques là où, du point de vue du sujet schizophrène (Figure 2), la cohérence sémantique est parfaitement préservée.

\section{Conclusion}

En développant une méthodologie d'analyse d'inspiration pragmatique et sémantique-formelle, nous avons montré comment l'usage du principe de charité pouvait être défendu dans un contexte radical comme celui de l'interprétation de la pensée schizophrène en conversation. Nous ne prétendons évidemment pas atteindre une compréhension au sens où nous partagerions la subjectivité du patient atteint de schizophrénie. L'approche linguistique défendue dans cet article offre cependant un aperçu de la rationalité propre au sujet schizophrène, qui est une dimension vraisemblablement primordiale dans la constitution de sa subjectivité.

La simple possibilité de construire deux représentations conversationnelles incompatibles indique bien qu'il y a du jeu dans la localisation de l'inconsistance chez les sujets schizophrènes. Nous aurions pu choisir de calquer la représentation conversationnelle du schizophrène sur celle du sujet normal. Cela aurait cependant produit une continuité entre les deux interlocuteurs, là où nous pensons qu'il n'y en a pas : ce qui se manifeste pour le sujet normal, qui relève de la pathologie du schizophrène, a toutes les chances de se manifester différemment pour ce dernier.

A partir du postulat selon lequel le sujet schizophrène est rationnel au sens le plus commun du terme, et qu'il se conforme aux normes de la logique tout autant que le sujet normal, l'application de notre méthodologie montre qu'il n'y a pas plus de contradictions dans la représentation sémantique d'un schizophrène que dans celle d'un sujet normal - même si du point de vue de ce 
dernier, il y a des contradictions (ce qui rejoint certains traits symptomatiques habituels de la pathologie). L'inconsistance semble être alors plutôt de nature pragmatique. Elle tient à un usage distinct de certaines procédures de gestion de l'échange chez les deux interlocuteurs. Notre analyse des conversations schizophrènes nous conduit ainsi à émettre l'hypothèse que le dysfonctionnement relève d'un déficit interactionnel, plutôt que d'une défaillance rationnelle. Cette hypothèse mériterait toutefois d'être étayée par une conception plus aboutie de la notion de " déficit interactionnel », ce que nous renvoyons à des travaux ultérieurs. 


\section{Références}

1 Quine, W.V.O., 1960: Word and Object, Cambridge, Mass, MIT Press. Trad. Fr. J. Dopp \& P. Gochet, 1977, Le mot et la chose, Paris, Flammarion.

2 Davidson, D., 1970: Mental events. Repr. in D. Davidson, 1980, Essays on Actions and Events, Oxford, Oxford University Press.

3 Laugier, S, 2003: Charité, traduction radicale et prélogicité, Revue de métaphysique et de morale, $\mathrm{n}^{\circ} 1 / 2003,63-83$.

4 Bonnay, D., Cozic, M., 2011: Principe de charité et sciences de l'homme, in Th. Martin (ed.), Les sciences humaines sont-elles des sciences?, Paris, Vuibert.

5 Sass, L. A., 1994: The paradoxes of delusion: Wittgenstein, Schreber, and the schizophrenic mind, New York, Cornell. Trad. Fr. P.-H. Castel, 2010, Les paradoxes du délire, Paris, Ithaque.

6 Sass, L. A., 2003: Incomprehensibility and Understanding: On the Interpretation of Severe Mental Illness, Philosophy, Psychiatry, \& Psychology, Volume 10, Number 2, June 2003, pp. 125-132

7 Henriksen, M. G., 2011: On incomprehensibility in schizophrenia, Phenom Cogn Sci, DOI 10.1007/s11097-010-9194-7

8 Priest, G., 2003: On Alternative Geometries, Arithmetics, and Logics; a Tribute to Łukasiewicz, Studia Logica 74, 441-468.

9 Łukasiewicz, J., 1910: O zasadzie sprzeczności u Arystotelesa. Trad. Fr. D. Sikora, 2000, Du principe de contradiction chez Aristote, Paris, L’Éclat.

10 Campbell, J, 2001: Rationality, meaning and the analysis of delusions, Philosophy, Psychiatry, and Psychology, 8, 89-100.

11 Villadsen, J., 2004: Paraconsistent Assertions, in G. Lindemann et al. (eds.): MATES 2004, LNAI 3187, Berlin Heidelberg, Springer, 99-113.

12 Brunet, J., 2010: Théorie du raisonnement et perspective de la première personne, Philosophiques, vol. 37, n², 411-437. 
13 Asher, N. Lascarides, A., 2003, Logics of Conversation, Cambridge University Press.

14 Kamp H., Reyle U., 1993: From Discourse to Logic, Dordrecht, Kluwer.

15 Roulet, E., et al., 1985: L'articulation du discours en français contemporain, Berne, Peter Lang.

16 Musiol M., Rebuschi M., 2007: La rationalité de l'incohérence en conversation schizophrène, Psychologie française, 52(2), 137-169.

17 Rebuschi, M., Musiol, M., 2010: Underspecification and pathological conversation : a S-DRT based formalization. In Journées de Sémantique Formelle. Nancy-Université LORIA-CNRS.

18 Amblard, M., Musiol, M., Rebuschi, M., 2010, An S-DRT based analysis of pathological dialogues, CAULD, nov. 2010.

19 Lascarides, A., Asher, N., 1993: Temporal interpretation, discourse relations and commonsense entailment, Linguistics and Philosophy 16: 437-493.

20 Musiol, M., Verhaegen, F, 2009: Appréhension et catégorisation de l'expression de la symptomatologie schizophrénique dans l'interaction verbale. Annales Médico-Psychologiques, 167 (10), 717-727.

21 Amblard, M., Musiol, M., Rebuschi, M.: "Une analyse basée sur la S-DRT pour la modélisation de dialogues pathologiques", Actes de la 18e conférence sur le Traitement Automatique des Langues Naturelles - TALN 2011, Montpellier, 27 juin-1 ${ }^{\text {er }}$ juillet 2011. 